OPEN ACCESS

Edited by:

John R. Lukens,

University of Virginia, United States

Reviewed by:

Christopher Lupfer,

Missouri State University,

United States

Prajwal Gurung,

University of lowa, United States

${ }^{*}$ Correspondence:

Sonia Villapol

svillapo/@houstonmethodist.org

orcid.org/0000-0002-6174-4113

†These authors have contributed equally to this work

Specialty section:

This article was submitted to

Multiple Sclerosis and

Neuroimmunology,

a section of the journal

Frontiers in Immunology

Received: 30 September 2018 Accepted: 09 November 2018 Published: 27 November 2018

Citation:

Treangen TJ, Wagner J, Burns MP and Villapol S (2018) Traumatic Brain Injury in Mice Induces Acute Bacterial

Dysbiosis Within the Fecal Microbiome. Front. Immunol. 9:2757

doi: 10.3389/fimmu.2018.02757

\section{Traumatic Brain Injury in Mice Induces Acute Bacterial Dysbiosis Within the Fecal Microbiome}

\author{
Todd J. Treangen ${ }^{1}$, Justin Wagner ${ }^{2}$, Mark P. Burns ${ }^{3 \dagger}$ and Sonia Villapol ${ }^{4 \star \dagger}$ \\ ${ }^{1}$ Department of Computer Science, Rice University, Houston, TX, United States, ${ }^{2}$ Center for Bioinformatics and \\ Computational Biology, University of Maryland, College Park, MD, United States, ${ }^{3}$ Department of Neuroscience, Georgetown \\ University, Washington, DC, United States, ${ }^{4}$ Center for Neuroregeneration, Houston Methodist Research Institute, Houston, \\ TX, United States
}

The secondary injury cascade that is activated following traumatic brain injury (TBI) induces responses from multiple physiological systems, including the immune system. These responses are not limited to the area of brain injury; they can also alter peripheral organs such as the intestinal tract. Gut microbiota play a role in the regulation of immune cell populations and microglia activation, and microbiome dysbiosis is implicated in immune dysregulation and behavioral abnormalities. However, changes to the gut microbiome induced after acute TBI remains largely unexplored. In this study, we have investigated the impact of TBI on bacterial dysbiosis. To test the hypothesis that $\mathrm{TBI}$ results in changes in microbiome composition, we performed controlled cortical impact (CCI) or sham injury in male 9-weeks old C57BL/6J mice. Fresh stool pellets were collected at baseline and at $24 \mathrm{~h}$ post-CCl. 16S rRNA based microbiome analysis was performed to identify differential abundance in bacteria at the genus and species level. In all baseline vs. $24 \mathrm{~h}$ post-CCl samples, we evaluated species-level differential abundances via clustered and annotated operational taxonomic units (OTU). At a high-level view, we observed significant changes in two genera after TBI, Marvinbryantia, and Clostridiales. At the species-level, we found significant decreases in three species (Lactobacillus gasseri, Ruminococcus flavefaciens, and Eubacterium ventriosum), and significant increases in two additional species (Eubacterium sulci, and Marvinbryantia formatexigens). These results pinpoint critical changes in the genus-level and species-level microbiome composition in injured mice compared to baseline; highlighting a previously unreported acute dysbiosis in the microbiome after TBI.

Keywords: microbiome, gut-brain axis, Lactobacillus, brain damage, gut microbes, traumatic brain injury, bacterial dysbiosis, controlled cortical impact injury

\section{INTRODUCTION}

Traumatic brain injury (TBI) is a major cause of death and disability that represents one of the most prevalent injury types sustained by the worldwide population (1). It is known to cause a massive neuronal loss and oxidative stress in the cortical region around the site of the impact formed $(2,3)$. Brain injuries also cause imbalances and gastrointestinal dysfunction (4). Specifically, gut barrier dysfunction results from high plasma levels of endotoxins and increased intestinal permeability following TBI (5), and evidence also points to an effect of commensal gut microbiota on the brain, now often referred to as the microbiome-gut-brain axis. 
The bidirectional influence of the gut-brain axis that modulates the neuroinflammatory process occurring at the time of the TBI and over hours, days, or weeks that follow. The gut microbiota can also influence brain function and behavior through the peripheral and central immune system, affecting to the circulating cytokines, which can act on corresponding receptors in neurons, glial cells, and endothelial cells to induce behavioral changes (6-8). As an example, the gut microbiome has emerged as a potent regulator of the immune system; inflammatory changes that occur after brain trauma are immediate and illustrate how cortical injury can lead to inflammatory consequences in the lining of the gastrointestinal mucosa (9). Gut microbiota likely plays a role in regulating intestinal barrier function that prevents the penetration of pathogenic compounds, and intestinal barrier dysfunction has been associated as a consequence of TBI (10). Healthy gut microbiota is critical for preventing bacterial translocation. Regulating diversity and balance in the healthy gut is still an active area of research, pioneered by the Human Microbiome Project (11) and MetaHIT $(12,13)$ projects that surveyed an extensive collection of healthy and unhealthy adults to get a better understanding of what it means to have a "healthy" gut microbiome (14).

Recently, the gastrointestinal system has been identified as being impacted by brain injury. Evidence supporting a bidirectional communication between the gut and brain includes changes to the mouse microbiome in animal models of brain ischemia (15) and spinal cord injury (14) and how intestinal dysbiosis alters immune homeostasis and injury recovery $(6,16)$. There is no data on the gut microbiome in TBI, however moderate to severe brain trauma in humans reduces gastric emptying (17) and intestinal contractility (18). Experimental TBI in mice caused a breakdown in the gut barrier with increased intestinal permeability, resulting in high levels of endotoxins $(5,19)$.

Changes to the gut microbiome represent a therapeutic avenue for the treatment of TBI due to the communication between injured brain and disruptions of the gut microbiome and its relation on the neuropathology of injury. In this study, we have analyzed microbial changes that occur $24 \mathrm{~h}$ after TBI in mice. Our results demonstrate that CCI causes a rapid shift in relative abundance of five species, including changes in the diversity of the psychoactive Lactobacillaceae family and protective Lachnospiraceae family, both commonly found in the human gut microbiome.

\section{METHODS}

\section{Mice and Controlled Cortical Impact Injury}

9-weeks-old male C57BL/6J mice were housed in a room maintained at a condition with $12 \mathrm{~h}$ light dark cycle, two mice per cage, eight mice per group. Daily fecal samples were pooled and redistributed amongst all of the experimental cages. Controlled Cortical Impact (CCI) injury was conducted as previously described (20). Sham mice received all procedures except contusion. Feces samples were obtained from each mouse temporarily housed individually in a sterile cage without bedding and collected in sterile tubes 1 day before and 1 day after CCI injury. Mice were euthanized with $\mathrm{CO}_{2}$ following the Institutional Animal Care and Use Committee (IACUC) a standard procedure for euthanasia. All animal experiments were performed in accordance with approved protocol \# 2017-0077 from Georgetown University Institutional Animal Care and Use Committee and comply with the approved institutional guidelines and regulations.

\section{Fecal DNA Extraction}

Genomic bacterial DNA was isolated from frozen stool samples using the QIAamp Fast DNA Stool Mini Kit (Qiagen). Snap-frozen fecal samples stored at $-80^{\circ} \mathrm{C}$ were added to sterile tubes and treated as described in the manufacturer's instructions. The tubes containing the pretreated samples were placed into a homogenizer and disrupted. The concentration of the extracted genomic DNA was qualified with Nanodrop 2000 UV spectrophotometer (Thermo Scientific).

\section{Sequencing of 16S rRNA V3-V4 Regions}

The gene-specific sequences used in this protocol target the $16 \mathrm{~S} \mathrm{V3}$ and V4 region. Sequencing libraries of the V3-V4 region were prepared according to the Illumina MiSeq system instructions. 16S bacterial rRNA gene were amplified using polymerase chain reaction (PCR) amplification with V3 and V4 region primers: (forward: 5' TCGTCGGCAGCGTCAGAT GTGTATAAGAGACAGCCTACGGGNGGCWGCAG; and reverse:5'GTCTCGTGGGCTCGGAGATGTGTATAAGAG ACAGGACTACHVGGGTATCTAATCC) for the first PCR and Nextera XT. Amplicons were generated using primers corresponding to the hypervariable regions, and the PCR products were purified. A library was created by targeting the $16 \mathrm{~S} \mathrm{V3}$ and V4 regions. Sequencing was performed via Illumina MiSeq platform using a 300 bp paired-end libraries.

\section{Operational Taxonomic Units (OTU) and Statistical Analyses}

The sequences generated from PCR-amplified 16S rRNA genes were quality inspected and filtered using Trimmomatic. We clustered the reads using DNAclust (21) into OTUs, normalized using Cumulative Sum Scaling, and assessed differential abundance with metagenomeSeq. QIIME2 was used to perform alpha and beta diversity analyses (qiime diversity core-metrics) and longitudinal (qiime longitudinal pairwise-differences) and volatility analyses (qiime longitudinal volatility). Log-fold change analysis and feature inspection were performed with MetaViz platform (22) and, interactive relative abundance plots were generated using Krona (23) and QIIME2 (24). Statistically significant differences in specieslevel relative abundance between paired samples were identified both using the non-parametric Wilcoxon ranked sum test and metagenomeSeq (25). 


\section{RESULTS}

\section{Genus-Level Changes After Traumatic Brain Injury}

We have characterized the mouse gut microbiome using $16 \mathrm{~S}$ rRNA analysis of multiple stool samples following experimental TBI. We found numerous bacterial species with significant $\log$ fold decreases in abundance $24 \mathrm{~h}$ post-TBI compared to either sham mice (baseline and $24 \mathrm{~h}$ ) or their baseline (Figure 1A). Specifically, the Lactobacillus genus relative abundance noticeably decreased, by up to a 2 log fold change, but this change was not statistically significant $(p>0.05)$.
However, Marvinbryantia $(p<0.05)$ and Clostridiales genera $(p<0.05)$ both significantly increase after TBI (Figure 1A). The Krona chart highlights the genus differential abundance in sham mice, TBI-baseline and $24 \mathrm{~h}$ post-TBI mice. TBI-baseline and sham exhibit similar compositions, while $24 \mathrm{~h}$ post-TBI Krona plot highlights a subtle shift in the community composition (Figure 1B).

\section{Species-Level Changes After Traumatic Brain Injury}

The QIIME species barplot (Figure 2A) shows the overall species-level relative abundance across both TBI and sham

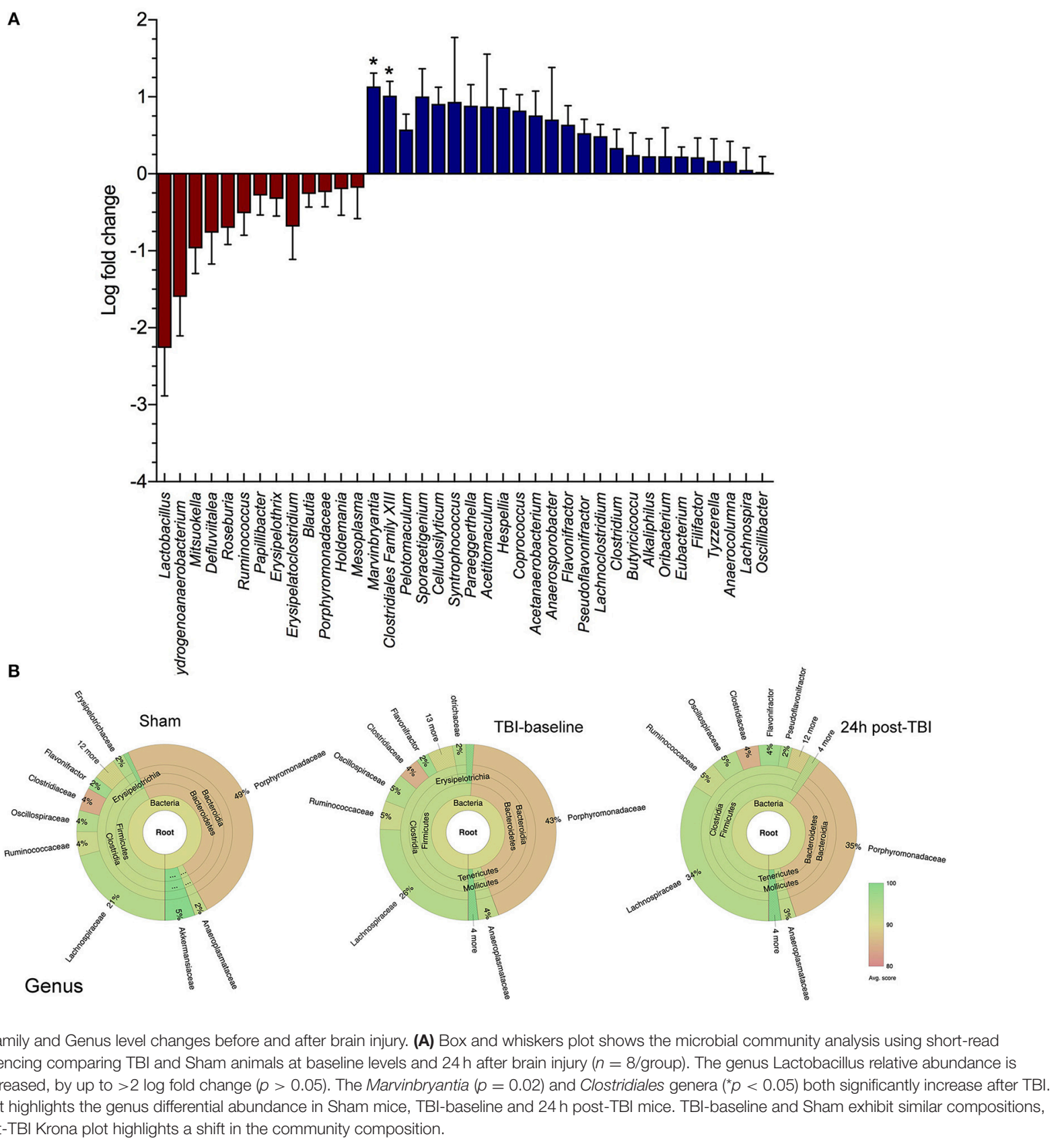




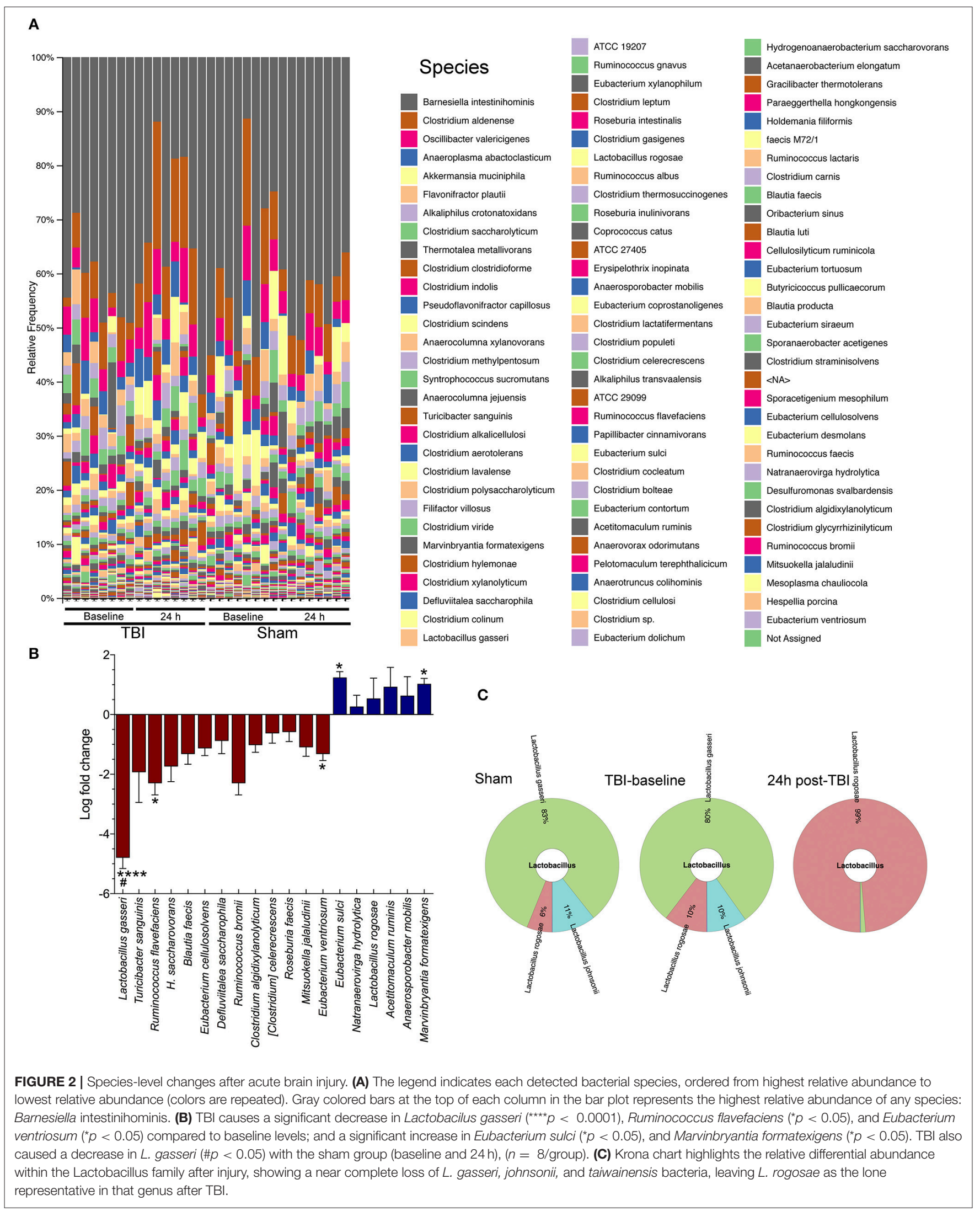


mice, at baseline and $24 \mathrm{~h}$, with little to no noticeable patterns in differential abundances across samples. However, when we compared TBI group basal samples to TBI-24h, we observed a significant change in four bacterial species (Figure 2B). Specifically, TBI caused a significant decrease in Lactobacillus gasseri $\left({ }^{* * * *} p<0.0001\right)$, Ruminococcus flavefaciens $\left({ }^{*} p<0.05\right)$, and Eubacterium ventriosum $(* p<0.05)$ compared to baseline levels; and a significant increase in Eubacterium sulci $\left({ }^{*} p<0.05\right)$, and Marvinbryantia formatexigens $(* p<0.05)$. TBI also caused a decrease in $L$. gasseri $\left({ }^{\#} p<0.05\right)$ with significant differences observed in the sham group, indicating a possible shared stress response. Lactobacillus gasseri after TBI decreased by more than a 4-fold log change compared to basal animals (metagenomeSeq) (Figure 2B). The Krona chart (Figure 2C) highlights the relative differential abundance within the Lactobacillus family after injury, showing a near complete loss of Lactobacillus gasseri, johnsonii, and taiwainensis bacteria, leaving L. rogosae as the lone representative in that genus after TBI. Specifically, L. gasseri is the predominant member of the Lactobacillus genus in sham and TBI-baseline mice, making up over $80 \%$ of the Lactobacillus population (Figure 2C).

\section{DISCUSSION}

In this study, we describe acute changes in the gut microbiome after brain damage in mice. Specifically, our findings suggest that specific commensal microbiota might play a role in the recovery from brain injury. Although, a link between TBI and intestinal dysfunction was previously demonstrated $(5,19)$, the focus of attention has shifted to gut microbiota as a critical factor in the inflammatory, immunological, or anxiety-related response and post-injury depression $(26,27)$.TBI causes a rapid shift in microbiota diversity within $24 \mathrm{~h}$ of brain injury, including a dramatic change in the diversity of the psychoactive Lactobacillus family (Figure 2). We found significant increases and decreases after TBI. Most of the identified differentially abundant species, including L. gasseri, M. formatexigens, and E. ventriosum, are known to inhabit the human gut microbiome (28), indicating translational potential. If follow up studies prove fruitful; these bacteria could potentially be translated to human TBI patients for improved neuroinflammatory and neurological recovery.

We also observed significant changes in multiple bacterial species. We found L. gasseri after TBI to decrease by a more than 4-fold log change compared to basal animals. Probiotic Lactobacillus strains have been associated with the attenuation of anxiety deficits $(29,30)$. Also, previous studies have demonstrated that Lactobacillus and Bifidobacterium species may prevent chronic psychological stress, reduce apoptosis in several brain regions, and improve learning and memory in mice $(29,31)$. Curiously, L. gasseri was also decreased in sham mice after $24 \mathrm{~h}$. This decrease could be a consequence of stress as it was previously described (32), due to the handling and anesthesia of the sham mice that cause stress. Ruminococcus flavieciens was also found to be decreased after brain injury. This bacterium is most commonly found in the rumen of wild and domesticated animals and has been previously associated with stress response (33). A decrease of Ruminococcus spp was previously documented in amyotrophic lateral sclerosis patients with signs of intestinal inflammation (34). Also, patients with Crohn's disease have been found to have lower levels of Ruminococcus albus than healthy individuals (35). We also found an increase of E. ventriosum in the injured mice. This bacterium was previously found to be increased in obese adults $(36,37)$ suggesting that gut microbiota composition is related to obesity. We did not find a correlation between the increased relative abundance of E. ventriosum and weight. Finally, our results also highlight an increase in the $M$. formatexigens species in mice after TBI. M. formatexigens consumes oligosaccharides, does not impact the redox state of the gut, and boosts the yield of succinate (38). Interestingly, succinate receptors have been found in the gut epithelium, representing a potential therapeutic target for the succinate toxicity (39). These receptors may mediate the local stress responses, including cerebral ischemia, hypoxia or TBI. A recent study using a rat model of TBI found phyla-level changes in alpha-diversity at $2 \mathrm{~h}$ postinjury (40). The small number of sham animals (4 per group) requiring follow-up studies to confirm these complementary findings.

Probiotics may confer a health benefit to the brain function and involved in the maintenance of the diversity of gut microbiota $(41,42)$. Our findings raise the intriguing possibility that probiotics may confer a health benefit to the brain following TBI. Data from animal models of stroke and spinal cord injury support the hypothesis that injury to the CNS causes a downstream effect on the gut microbiome (15) and treatments targeting biodiversity in the gut can impact CNS injury outcome $(6,16)$. Though not yet explored for TBI, probiotic treatment has been shown to affect the immune response by altering the Th1/Th2 imbalance (43).

In summary, the role of microbes in gut-brain cross-talk and the pathology of brain trauma represents a therapeutic target for recovery from TBI. Based on these preliminary findings, we hypothesize that repairing gut dysbiosis caused by TBI using probiotic treatments might serve as a new tool for reducing brain inflammation and improving anxiety and depression phenotypes after TBI. Future follow-up studies will be required to expand on research avenues indicated by our initial findings.

\section{ETHICS STATEMENT}

All procedures were performed in accordance with protocols approved by the Georgetown University Animal Care and Use Committee.

\section{AUTHOR CONTRIBUTIONS}

TT, JW, MB, and SV conducted all the experiments. MB and SV performed the animal surgery, and SV performed bacterial DNA purification. TT and JW performed the bioinformatic and statistical analyses. TT and SV designed the study, analyzed the data, interpreted the results, and wrote the manuscript. All authors contributed to the editing of the manuscript. All the authors read and approved the final version before submission. 


\section{FUNDING}

This work was supported by an R21NS106640 (SV) grant from the National Institute for Neurological Disorders and Stroke (NINDS), and funds from the Houston Methodist Research Institute.

\section{REFERENCES}

1. Dewan MC, Rattani A, Gupta S, Baticulon RE, Hung YC, Punchak M, et al. Estimating the global incidence of traumatic brain injury. J Neurosurg. (2018) doi: 10.3171/2017.10.JNS17352. [Epub ahead of print].

2. Abdul-Muneer PM, Chandra N, Haorah J. Interactions of oxidative stress and neurovascular inflammation in the pathogenesis of traumatic brain injury. Mol Neurobiol. (2015) 51:966-79. doi: 10.1007/s12035-014-8752-3

3. Villapol S, Acarin L, Faiz M, Castellano B, Gonzalez B. Distinct spatial and temporal activation of caspase pathways in neurons and glial cells after excitotoxic damage to the immature rat brain. J Neurosci Res. (2007) 85:354556. doi: 10.1002/jnr.21450

4. Sundman MH, Chen NK, Subbian V, Chou YH. The bidirectional gutbrain-microbiota axis as a potential nexus between traumatic brain injury, inflammation, and disease. Brain Behav Immun. (2017) 66:31-44. doi: 10.1016/j.bbi.2017.05.009

5. Hang CH, Shi JX, Li JS, Wu W, Yin HX. Alterations of intestinal mucosa structure and barrier function following traumatic brain injury in rats. World J Gastroenterol. (2003) 9:2776-81. doi: 10.3748/wjg.v9.i12.2776

6. Benakis C, Brea D, Caballero S, Faraco G, Moore J, Murphy M, et al. Commensal microbiota affects ischemic stroke outcome by regulating intestinal gammadelta T cells. Nat Med. (2016) 22:516-23. doi: $10.1038 / \mathrm{nm} .4068$

7. Luczynski P, McVey Neufeld KA, Oriach CS, Clarke G, Dinan TG, Cryan JF. Growing up in a bubble: using germ-free animals to assess the influence of the gut microbiota on brain and behavior. Int J Neuropsychopharmacol. (2016) 19:pyw020. doi: 10.1093/ijnp/pyw020

8. Pigrau M, Rodino-Janeiro BK, Casado-Bedmar M, Lobo B, Vicario M, Santos J, et al. The joint power of sex and stress to modulate brain-gut-microbiota axis and intestinal barrier homeostasis: implications for irritable bowel syndrome. Neurogastroenterol Motil. (2016) 28:463-86. doi: 10.1111/nmo.12717

9. Bansal V, Costantini T, Ryu SY, Peterson C, Loomis W, Putnam J, et al. Stimulating the central nervous system to prevent intestinal dysfunction after traumatic brain injury. J Trauma. (2010) 68:1059-64. doi: 10.1097/TA.0b013e3181d87373

10. Li H, Sun J, Du J, Wang F, Fang R, Yu C, et al. Clostridium butyricum exerts a neuroprotective effect in a mouse model of traumatic brain injury via the gut-brain axis. Neurogastroenterol Motil. (2018) 30:e13260. doi: $10.1111 / \mathrm{nmo} .13260$

11. Human Microbiome Project C. Structure, function and diversity of the healthy human microbiome. Nature (2012) 486:207-14. doi: 10.1038/nature11234

12. Koren O, Knights D, Gonzalez A, Waldron L, Segata N, Knight R, et al. A guide to enterotypes across the human body: meta-analysis of microbial community structures in human microbiome datasets. PLoS Comput Biol. (2013) 9:e1002863. doi: 10.1371/journal.pcbi.1002863

13. Morgan XC, Segata N, Huttenhower C. Biodiversity and functional genomics in the human microbiome. Trends Genet. (2013) 29:51-8. doi: 10.1016/j.tig.2012.09.005

14. Manrique P, Bolduc B, Walk ST, van der Oost J, de Vos WM, Young MJ. Healthy human gut phageome. Proc Natl Acad Sci USA. (2016) 113:10400-5. doi: 10.1073/pnas.1601060113

15. Singh V, Roth S, Llovera G, Sadler R, Garzetti D, Stecher B, et al. Microbiota dysbiosis controls the neuroinflammatory response after stroke. J Neurosci. (2016) 36:7428-40. doi: 10.1523/JNEUROSCI.1114-16.2016

16. Kigerl KA, Hall JC, Wang L, Mo X, Yu Z, Popovich PG. Gut dysbiosis impairs recovery after spinal cord injury. J Exp Med. (2016) 213:2603-20. doi: $10.1084 /$ jem. 20151345

\section{ACKNOWLEDGMENTS}

We acknowledge the support from GCB Sequencing and Genomic Technologies laboratory staff of Duke University for conducting the library construction, and sequencing of collected samples.

17. Kao $\mathrm{CH}$, ChangLai SP, Chieng PU, Yen TC. Gastric emptying in head-injured patients. Am J Gastroenterol. (1998) 93:1108-12. doi: 10.1111/j.1572-0241.1998.00338.x

18. Olsen AB, Hetz RA, Xue H, Aroom KR, Bhattarai D, Johnson E, et al. Effects of traumatic brain injury on intestinal contractility. Neurogastroenterol Motil. (2013) 25:593-e463. doi: 10.1111/nmo.12121

19. Ma EL, Smith AD, Desai N, Cheung L, Hanscom M, Stoica BA, et al. Bidirectional brain-gut interactions and chronic pathological changes after traumatic brain injury in mice. Brain Behav Immun. (2017) 66:56-69. doi: 10.1016/j.bbi.2017.06.018

20. Villapol S, Loane DJ, Burns MP. Sexual dimorphism in the inflammatory response to traumatic brain injury. Glia (2017) 65:1423-38. doi: 10.1002/glia.23171

21. Ghodsi M, Liu B, Pop M. DNACLUST: accurate and efficient clustering of phylogenetic marker genes. BMC Bioinformatics (2011) 12:271. doi: 10.1186/1471-2105-12-271

22. Wagner J, Chelaru F, Kancherla J, Paulson JN, Zhang A, Felix V, et al. Metaviz: interactive statistical and visual analysis of metagenomic data. Nucleic Acids Res. (2018) 46:2777-87. doi: 10.1093/nar/gky136

23. Ondov $\mathrm{BD}$, Bergman $\mathrm{NH}$, Phillippy $\mathrm{AM}$. Interactive metagenomic visualization in a Web browser. BMC Bioinformatics (2011) 12:385. doi: 10.1186/1471-2105-12-385

24. Caporaso JG, Kuczynski J, Stombaugh J, Bittinger K, Bushman FD, Costello EK, et al. QIIME allows analysis of high-throughput community sequencing data. Nat Methods (2010) 7:335-6. doi: 10.1038/nmeth.f.303

25. Paulson JN, Stine OC, Bravo HC, Pop M. Differential abundance analysis for microbial marker-gene surveys. Nat Methods (2013) 10:1200-2. doi: 10.1038/nmeth.2658

26. Kelly JR, Borre Y, O' Brien C, Patterson E, El Aidy S, Deane J, et al. Transferring the blues: depression-associated gut microbiota induces neurobehavioural changes in the rat. J Psychiatr Res. (2016) 82:109-18. doi: 10.1016/j.jpsychires.2016.07.019

27. Robertson RC, Seira Oriach C, Murphy K, Moloney GM, Cryan JF, Dinan TG, et al. Omega-3 polyunsaturated fatty acids critically regulate behaviour and gut microbiota development in adolescence and adulthood. Brain Behav Immun. (2017) 59:21-37. doi: 10.1016/j.bbi.2016. 07.145

28. Lepage P, Leclerc MC, Joossens M, Mondot S, Blottiere HM, Raes J, et al. A metagenomic insight into our gut's microbiome. Gut (2013) 62:146-58. doi: 10.1136/gutjnl-2011-301805

29. Messaoudi M, Violle N, Bisson JF, Desor D, Javelot H, Rougeot C. Beneficial psychological effects of a probiotic formulation (Lactobacillus helveticus R0052 and Bifidobacterium longum R0175) in healthy human volunteers. Gut Microbes (2011) 2:256-61. doi: 10.4161/gmic.2.4.16108

30. Zakostelska Z, Kverka M, Klimesova K, Rossmann P, Mrazek J, Kopecny J, et al. Lysate of probiotic Lactobacillus casei DN-114 001 ameliorates colitis by strengthening the gut barrier function and changing the gut microenvironment. PLoS ONE (2011) 6:e27961. doi: 10.1371/journal.pone.0027961

31. Messaoudi M, Lalonde R, Violle N, Javelot H, Desor D, Nejdi A, et al. Assessment of psychotropic-like properties of a probiotic formulation (Lactobacillus helveticus R0052 and Bifidobacterium longum R0175) in rats and human subjects. Br J Nutr. (2011) 105:755-64. doi: 10.1017/S0007114510004319

32. Marin IA, Goertz JE, Ren T, Rich SS, Onengut-Gumuscu S, Farber E, et al. Microbiota alteration is associated with the development of stress-induced despair behavior. Sci Rep. (2017) 7:43859. doi: 10.1038/srep43859 
33. Park J, Lee J, Yeom Z, Heo D, Lim YH. Neuroprotective effect of Ruminococcus albus on oxidatively stressed SH-SY5Y cells and animals. Sci Rep. (2017) 7:14520. doi: 10.1038/s41598-017-15163-5

34. Rowin J, Xia Y, Jung B, Sun J. Gut inflammation and dysbiosis in human motor neuron disease. Physiol Rep. (2017) 5:e13443. doi: 10.14814/phy2.13443

35. Kang S, Denman SE, Morrison M, Yu Z, Dore J, Leclerc M, et al. Dysbiosis of fecal microbiota in Crohn's disease patients as revealed by a custom phylogenetic microarray. Inflamm Bowel Dis. (2010) 16:2034-42. doi: 10.1002/ibd.21319

36. Druart C, Dewulf EM, Cani PD, Neyrinck AM, Thissen JP, Delzenne NM. Gut microbial metabolites of polyunsaturated fatty acids correlate with specific fecal bacteria and serum markers of metabolic syndrome in obese women. Lipids (2014) 49:397-402. doi: 10.1007/s11745-014-3881-Z

37. Kasai C, Sugimoto K, Moritani I, Tanaka J, Oya Y, Inoue H, et al. Comparison of the gut microbiota composition between obese and non-obese individuals in a Japanese population, as analyzed by terminal restriction fragment length polymorphism and next-generation sequencing. BMC Gastroenterol. (2015) 15:100. doi: 10.1186/s12876-0150330-2

38. Rey FE, Faith JJ, Bain J, Muehlbauer MJ, Stevens RD, Newgard CB, et al. Dissecting the in vivo metabolic potential of two human gut acetogens. J Biol Chem. (2010) 285:22082-90. doi: 10.1074/jbc.M110.117713

39. Ariza AC, Deen PM, Robben JH. The succinate receptor as a novel therapeutic target for oxidative and metabolic stress-related conditions. Front Endocrinol. (2012) 3:22. doi: 10.3389/fendo.2012.00022
40. Nicholson SE, Watts LT, Burmeister DM, Merrill D, Scroggins S, Zou Y, et al. Moderate traumatic brain injury alters the gastrointestinal microbiome in a time-dependent manner. Shock (2018). doi: 10.1097/SHK.0000000000001211. [Epub ahead of print].

41. Sirisinha S. The potential impact of gut microbiota on your health: Current status and future challenges. Asian Pac J Allergy Immunol. (2016) 34:249-64. doi: 10.12932/AP0803

42. Bienenstock J, Kunze W, Forsythe P. Microbiota and the gut-brain axis. Nutr Rev. (2015) 73 (Suppl 1):28-31. doi: 10.1093/nutrit/nuv019

43. Tan M, Zhu JC, Du J, Zhang LM, Yin HH. Effects of probiotics on serum levels of Th1/Th2 cytokine and clinical outcomes in severe traumatic brain-injured patients: a prospective randomized pilot study. Crit Care (2011) 15:R290. doi: $10.1186 /$ cc10579

Conflict of Interest Statement: The authors declare that the research was conducted in the absence of any commercial or financial relationships that could be construed as a potential conflict of interest.

Copyright (c) 2018 Treangen, Wagner, Burns and Villapol. This is an open-access article distributed under the terms of the Creative Commons Attribution License (CC $B Y)$. The use, distribution or reproduction in other forums is permitted, provided the original author(s) and the copyright owner(s) are credited and that the original publication in this journal is cited, in accordance with accepted academic practice. No use, distribution or reproduction is permitted which does not comply with these terms. 